\title{
Intravesical Treatments of Bladder Cancer: Review
}

\author{
Zancong Shen, ${ }^{1}$ Tong Shen, ${ }^{1}$ M. Guillaume Wientjes, ${ }^{1,2}$ Michael A. O'Donnell, ${ }^{3}$ and Jessie L.-S. Au ${ }^{1,2,4}$
}

Received February 2, 2008; accepted March 6, 2008; published online March 28, 2008

\begin{abstract}
For bladder cancer, intravesical chemo/immunotherapy is widely used as adjuvant therapies after surgical transurethal resection, while systemic therapy is typically reserved for higher stage, muscleinvading, or metastatic diseases. The goal of intravesical therapy is to eradicate existing or residual tumors through direct cytoablation or immunostimulation. The unique properties of the urinary bladder render it a fertile ground for evaluating additional novel experimental approaches to regional therapy, including iontophoresis/electrophoresis, local hyperthermia, co-administration of permeation enhancers, bioadhesive carriers, magnetic-targeted particles and gene therapy. Furthermore, due to its unique anatomical properties, the drug concentration-time profiles in various layers of bladder tissues during and after intravesical therapy can be described by mathematical models comprised of drug disposition and transport kinetic parameters. The drug delivery data, in turn, can be combined with the effective drug exposure to infer treatment efficacy and thereby assists the selection of optimal regimens. To our knowledge, intravesical therapy of bladder cancer represents the first example where computational pharmacological approach was used to design, and successfully predicted the outcome of, a randomized phase III trial (using mitomycin C). This review summarizes the pharmacological principles and the current status of intravesical therapy, and the application of computation to optimize the drug delivery to target sites and the treatment efficacy.
\end{abstract}

KEY WORDS: bladder cancer; computational modeling; intravesical therapy; pharmacokinetic/ pharmacodynamic; regional therapy.

\section{INTRODUCTION}

Bladder cancer is the fifth most common cancer in the United States, with an estimated 67,160 newly diagnosed cases and 13,750 deaths in the United States in 2007. The 5 -year survival rate is $82 \%$ for all stages combined (1). The standard of treatment for patients with superficial bladder cancer is surgical transurethal resection (TUR) of tumors, with an $80 \%$ early success rate. However, nearly $70 \%$ of these patients will develop tumor recurrence, with $25 \%$ showing

\footnotetext{
${ }^{1}$ College of Pharmacy, The Ohio State University, 500 West 12th Avenue, Columbus, Ohio 43210, USA.

${ }^{2}$ James Cancer Hospital and Solove Research Institute, The Ohio State University, 500 West 12th Avenue, Columbus, Ohio 43210, USA.

${ }^{3}$ Department of Urology, College of Medicine, University of Iowa, Iowa City, Iowa 52242, USA.

${ }^{4}$ To whom correspondence should be addressed. (e-mail: au.1@osu. edu)

ABBREVIATIONS: BCG, Bacille Calmette-Guérin; CAR, coxsackie/adenovirus receptor; CIS, carcinoma in situ; DMSO, dimethyl sulfoxide; GAG, glycosaminoglycans; GM-CSF, granulocyte-macrophage colony stimulating factor; IFN, interferons; IL-2, interleukin-2; IL-12, interleukin-12; KLH, keyhole limpet hemocyanin; MMC, mitomycin C; PLK-1, polo-like kinase-1; PPA, pipemidic acid; rVV, recombinant vaccinia virus; siRNA, small interfering RNA; TNF, tumor necrosis factor; TUR, transurethal resection.
}

progression to muscle-invading disease, within 5 years with TUR (2). Intravesical chemotherapy and immunotherapy are widely used as adjuvant therapies after TUR, to prevent recurrence and progression of superficial disease. Systemic therapy is typically reserved for higher stage, muscle-invading, or metastatic diseases.

The urinary bladder is an ideal organ for regional therapy. The urethra provides easy access of therapeutic agents to the urinary bladder. The presence of the specialized asymmetric unit membrane on the urothelium serves as a barrier and limits the absorption of molecules or particulates into the systemic circulation. For most small molecule drugs, less than $5 \%$ of the dose is absorbed into the systemic circulation (3-5). The rationale for intravesical therapy is to maximize the exposure of tumors located in the bladder cavity to therapeutics agents while limiting the systemic exposure and thereby limiting the host toxicities; the primary goal is to eradicate existing or residual tumors through direct cytoablation or immunostimulation. The unique properties of the urinary bladder render it a fertile ground for evaluating novel experimental approaches to regional therapy, including iontophoresis/electrophoresis, local hyperthermia, co-administration of permeation enhancers, bioadhesive carriers, magnetic-targeted particles and gene therapy.

This review consists of five parts. Part I summarizes the current status of intravesical treatments of bladder cancer. Part II discusses the drug disposition in bladder tissues during intravesical therapy. Part III discusses the application of 
computation to optimize intravesical treatments. Part IV summarizes the experimental approaches under preclinical and clinical evaluation. Part V provides the general perspectives.

\section{PART I. CURRENT STATUS OF INTRAVESICAL TREATMENTS}

Immunomodulators. Bacille Calmette-Guérin (BCG) is the most adopted first-line immunotherapeutic and is the most effective treatment for prophylaxis and treatment of carcinoma in situ (CIS). Phase I and II trials have shown that other immunoregulators such as interferons (IFN) (6-8), interleukin2 (IL-2) (9-11), interleukin-12 (IL-12) $(12,13)$, tumor necrosis factor (TNF) (14-16), keyhole limpet hemocyanin (KLH) (17, $18)$ and rubratin $(19,20)$ have activity in BCG-refractory patients, albeit with low durable remissions $(<20 \%)$.

Chemotherapy. Multiple chemotherapeutic agents, such as mitomycin C (MMC) (21-25), doxorubicin (26-28), epirubicin (29-32), thiotepa $(33,34)$, ethoglucid (35-37), valrubicin (38-40), cisplatin (41-43), gemcitabine (44-46), suramin $(47,48)$ or their combinations $(49-52)$ have been evaluated in patients; meta-analyses did not show apparent superiority of a particular treatment. The addition of intravesical chemotherapy to TUR yields, on average, a further reduction of recurrence by $14-17 \%$, but has limited benefits against disease progression (53).

$B C G$ vs chemotherapy. Compared to several chemotherapeutic agents (thiotepa, epirubicin and doxorubicin), BCG is more effective in preventing tumor recurrence (14-47\% lower recurrence rate) and disease progression (54). In patients with stage Ta or T1 carcinoma, BCG showed a lower recurrence rate $(38.6 \%$ versus $46.4 \%)$ and lower disease progression (34\% lower), compared to standard albeit suboptimal MMC treatments (i.e., no pharmacological interventions to improve the MMC delivery to tumor cells, see Part III) (55). Patients who failed BCG and desired avoiding radical cystectomy have been treated with intravesical IFN- $\alpha$ (56-59), valrubicin $(60)$ or gemcitabine $(61,62)$ with some limited success.

\section{PART II. DRUG DISPOSITION DURING INTRAVESICAL THERAPY}

Drug disposition in the bladder during intravesical therapy is affected by several attributes, i.e., physicochemical properties of the drug (molecular weight, hydrophilicity or lipophilicity, water/lipid partition coefficient), urine volume and $\mathrm{pH}$, patient hydration status, and integrity of urothelium. Our laboratory has provided the first pharmacokinetic models to describe drug disposition in urine and bladder tissues. These models enable the prediction of changes in drug concentration in different parts of bladder wall as a function of physiological, pathological or pharmacological parameters (63).
The first set of equations describes the urine pharmacokinetics during treatment:

$$
C_{\mathrm{u}}=\frac{\text { Dose }}{V_{\mathrm{u}}} e^{-\left(K_{\mathrm{a}}+K_{\mathrm{d}}\right) t}
$$

$$
V_{u}=V_{0}+K_{0} t+V_{r e s}
$$

where $C_{\mathrm{u}}$ is the urine drug concentration at time $t, V_{\mathrm{u}}$ is the volume of the urine, $K_{\mathrm{a}}$ is the first order rate constant describing drug absorption into the systemic circulation, $K_{\mathrm{d}}$ is the hybridized first order rate constant describing degradation, metabolism, and tissue binding, $V_{\mathrm{o}}$ is the dosing volume, $K_{0}$ is the zero order rate constant describing urine production, and $V_{\text {res }}$ is the post-catheterization residual urine volume.

The urine pharmacokinetic model provides the tool to depict changes in urine drug concentrations due to changes in physiological parameters that can vary from patient to patient (e.g., residual urine volume, urine $\mathrm{pH}$, urine production rate) and changes in drug-related parameters (e.g., dose, dosing volume, degradation in acidic or basic environment). It is noted that most of the small molecule drugs used in intravesical chemotherapy have $\mathrm{pH}$-dependent stability, e.g., MMC is unstable in $\mathrm{pH}<5$ or $\mathrm{pH}>8(64,65)$, thiotepa and its active metabolite are unstable in acidic $\mathrm{pH}(<5)$ and stable at alkaline $\mathrm{pH}(8.4)(66,67)$, whereas doxorubicin is more stable in acidic $\mathrm{pH}$ (5.4) than in alkaline $\mathrm{pH}$ (8.1) (68). $\mathrm{pH}$ also affects the antitumor activity, e.g., MMC is more active at acidic $\mathrm{pH}$ in monolayer cultures (but no $\mathrm{pH}$ dependent-effect in 3-dimensional cultures) (69), epirubicin is more active at alkaline $\mathrm{pH}$ (8.0) than at lower $\mathrm{pH}$ (6.0) (70). The urine production rate and residual urine volume can be altered by patients' hydration status, e.g., dehydration for $6 \mathrm{hr}$ decreased the average volume of urine production from 209 to $143 \mathrm{ml}$, which is further reduced to $103 \mathrm{ml}$ by co-administering an antidiuretic desmopressin (71).

For a drug-of-interest, the urine pharmacokinetic model can be established by defining the values of the rate constants in the equations. The values for the physiological parameters in patients can be readily obtained from the literature. The values for the drug-related parameters are either defined by investigators (e.g., dose, dosing volume) or by measuring the degradation rate in buffers with different $\mathrm{pH}$ values. Successful development of the urine pharmacokinetic model for a drug-of-interest would enable the prediction of the effects of changing the various parameters on the urine drug concentrations and, because the urine concentration is the driving force for delivering the drug to bladder tissues, on the drug concentrations in the urothelium layer.

The second set of equations describes the drug transport in bladder tissues, as a function of time and distance from the urine compartment. For this purpose, the bladder wall is 
divided into two sections, the urothelium (mucosa) that is not blood-perfused and the submucosal and muscle layers of the bladder that contain blood vessels and lymphatics. Drug transport from the urine compartment across the urothelium (about 7-10 cell layer or $200 \mu \mathrm{m}$ thick in man (72)) is depicted by diffusion across a single homogeneous diffusion barrier and is described by Fick's first law (Eq. 3). Drug transport across the submucosa and superficial muscle $(200-4,000 \mu \mathrm{m})$ is described by the distributed model (Eq. 4) $(5,63,73,74)$. The distributed model integrates drug diffusion through a porous structure and drug removal/absorption through the blood vessels.

Urothelium $(0-200 \mu \mathrm{m})$ :

$$
C_{\text {depth }}=C_{u}-\frac{C_{u}-C_{\text {uro }}}{200} \times \text { depth }
$$

Submucosa/superficial muscle layer (200-4,000 $\mu \mathrm{m})$ :

$$
C_{\mathrm{depth}}=\left(C_{\mathrm{uro}}-C_{\mathrm{b}}\right) \times e^{-\frac{0.693}{W_{1 / 2}} \times(\text { depth }-200)}+C_{\mathrm{b}}
$$

In the urothelium, $C_{\mathrm{u}}$ is the concentration of unionized drug in the bladder cavity and is the link between the urine and tissue pharmacokinetics. $C_{\text {uro }}$ is the concentration at the interface between the urothelium and the submucosa, or about $200 \mu \mathrm{m}$ away from the surface of the urothelium lining. $C_{\text {depth }}$ is the concentration at a particular tissue depth. In the urothelium, $C_{\text {depth }}$ declines linearly with increasing depth because diffusion is not dependent on the depth. In the submucosa and superficial muscle layer, $C_{\text {depth }}$ is determined by $C_{\text {uro }}$, the plasma drug concentration $C_{\mathrm{b}}$ in blood vessels, the tissue depth away from the urothelium and the half-width $\mathrm{W}_{1 / 2}$. The latter is the thickness of tissue over which the drug concentration declines by $50 \%$. Because the number of vessels encountered by the drug molecule increases with increasing tissue depth (assumed to be evenly distributed), the decline of $C_{\text {depth }}$ in submucosa and superficial muscle layers is first order with respect to tissue depth.

We have experimentally determined the pharmacokinetic model parameters for several drugs, i.e., MMC, doxorubicin, 5-fluorouridine and paclitaxel. The first three less lipophilic drugs show comparable penetration across the urothelium with a $C_{\mathrm{uro}} / C_{\mathrm{u}}$ ratio of 0.02 to 0.03 , whereas the more lipophilic paclitaxel shows a significantly higher $C_{\mathrm{uro}} / C_{\mathrm{u}}$ ratio of about 0.5 . These findings, together with the earlier finding of the extensive systemic absorption of the lipophilic small molecule thiotepa (MW, $189 \mathrm{Da})$ (75), indicate that lipophilicity is a key determinant of drug penetration across the urothelium. Another consideration is systemic absorption; the absorption of MMC, doxorubicin, 5-fluorouridine and paclitaxel after intravesical therapy is consistently lower compared to thiotepa $(<5 \%$ vs $20 \%)(3,4,73,76)$. We infer that an ideal drug for intravesical therapy would be a lipophilic compound that can readily penetrate the urothelium and remain localized in bladder tissue (as opposed to being rapidly absorbed into the systemic circulation). Note that the model for drug transport across the urothelium enables the computation of effects of altering the urothelium barrier function (e.g., compromised urothelium due to surgery or treatment with surfactant or absorption enhancers).

A second generation bladder tissue kinetic model was recently described (77). The major modification is the arbitrary subdivision of the bladder wall into 30 serially interconnected compartments (4 within the urothelium and 26 within the deeper, capillary-perfused tissues). This model was used to generate simulations of the concentration-depth profiles of a hydrophilic drug pipemidic acid (PPA), for the purpose of studying its systemic absorption rate, and the effects of co-administering bioadhesive permeation enhancers (chitosan and polycarpophil). The model parameters such as tissue partition coefficient and diffusion rate constants were obtained using in vitro drug transport data generated using the isolated porcine bladder. The mathematical expansion of the bladder tissues from 2 compartments into 30 compartments enables the inclusion of inter-compartmental drug transfer and drug absorption from each sub-compartment (e.g., different transport rates at different tissue locations) and thereby enhances the data fitting capability. It is noted, however, the anatomical and physiological relevance of the 30 sub-compartments is unclear and the model performance under in vivo conditions cannot be ascertained because the model parameters were obtained under in vitro conditions, i.e., without intact blood perfusion.

The above urine and tissue pharmacokinetic models provide the basis for computing drug delivery to the targeted, tumor-residing sites in the bladder as a function of treatment conditions (e.g., dose, drug concentration, volume of dosing solution, patient hydration status, treatment duration), during intravesical therapy. Integration of drug delivery with pharmacodynamic data such as the effective drug concentrations in preclinical models provides a means to rational design of intravesical treatments (see Part III).

\section{PART III. APPLICATION OF COMPUTATIONAL MODELING TO OPTIMIZE INTRAVESICAL TREATMENTS}

Response of a patient to a treatment is largely determined by whether his/her tumor cells are sensitive to the selected chemotherapy and whether sufficient drug is delivered to tumor cells. Tumor sensitivity is a biological/genetic property that cannot be readily controlled or manipulated. On the other hand, as discussed in Part II, the delivery of a therapeutic agent to tumor cells located in different parts of bladder wall can be depicted as mathematical relationships with controllable variables such as dose, volume of dosing solution, urine production rate during treatment, residual 
urine volume at the time of dose instillation, and urine $\mathrm{pH}$. Drug concentration in the urine compartment is directly affected by the first four variables (e.g., a lower dose or a larger urine volume diminishes the drug concentration), whereas the $\mathrm{pH}$ affects the drug stability and thereby indirectly determines the concentration of the intact drug. The following example demonstrates the application of computational modeling to clinical trial design (please see reference (63) for a more detailed discussion). First, we used the urine pharmacokinetic model described in Equations 1 to 4 to evaluate how changes in the variables or treatment conditions alter the drug delivery (63). The simulated drug amounts, equivalent to (concentration) $\mathrm{x}$ (treatment time) product or CxT, were then compared to the drug concentrations found active in patient bladder tumors $(n>60)$, in order to determine the fraction of patients likely to receive sufficient drug amount to produce a therapeutically meaningful response under various treatment conditions. Computation was used to evaluate seven changes in treatment conditions, including increasing the dose from 20 to $40 \mathrm{mg}$ or to $60 \mathrm{mg}$, decreasing the dosing volume from 40 to $20 \mathrm{ml}$, increasing instillation time from 120 to 240 minutes, decreasing urine production rate from 1.48 to $0.63 \mathrm{ml} / \mathrm{min}$, reducing residual volume from $32.4 \mathrm{ml}$ to $0 \mathrm{ml}$ and increasing $\mathrm{pH}$ from 5 to 7 . The simulations predicted that changes of the above parameters would affect the treatment outcome in the following rank order: dose $>$ urine residual volume $>$ urine production rate $>$ dosing volume $>$ urine $\mathrm{pH}>$ instillation time.

The three major findings of the computer simulation results are as follows. (a) Changing one parameter at a time would yield small incremental improvements (i.e., $<8 \%$ higher 13-month recurrence free rate) such that at least 450 patients would be required to demonstrate the benefit of changing a single individual treatment parameter. This finding suggests that the inconclusive results for the earlier trials that attempted to evaluate the benefits of changing various individual treatment parameters were likely due to inadequate patient sample size. (b) In contrast, simultaneous changes in five treatment parameters (see below) would increase the 13 -month recurrence rate by $18-20 \%$, a difference that is large enough to be detected with a relatively small number of patients (230 patients showing 116 events). (c) The simulation results further showed that two additional changes in treatment parameters (using the maximally tolerated dose of $60 \mathrm{mg}$ or increasing the treatment time from 2 to $4 \mathrm{hr}$ ) would not produce additional benefits.

The computer simulation results were used to synthesize an optimized MMC treatment protocol that was subsequently tested in a multi-center, two-arm phase III clinical trial (78). In this trial, superficial bladder cancer patients with histologically proven transitional cell carcinoma (Ta, T1, CIS) and at high risk for recurrence were randomized to optimized or standard treatment arms. Patients in the optimized arm received 40-mg dose of MMC, with pharmacological manipulations to maximize the drug delivery (ultrasound-guided bladder emptying, voluntary dehydration, urine alkalinization by oral sodium bicarbonate). In the standard arm, patients received a $20-\mathrm{mg}$ dose and $20 \mathrm{ml}$ dosing volume without manipulations. All patients received intravesical treatments weekly for 6 weeks.
The primary endpoint was time to recurrence and the secondary endpoint was recurrence-free rate. The results showed that in evaluable patients, the median time to recurrence was 29.1 months in the optimized arm, significantly higher than that in standard arm (11.8 months) $(\mathrm{p}<0.001)$. The projected 5-year recurrence-free rate with a median 11.7 months follow-up was $42.6 \%$ in optimized arm and $23.5 \%$ in standard arm, or a $19.1 \%$ increase. The extent of improvement is identical to the simulated prediction (18-20\% increase). To our knowledge, this is the first efficacy trial designed based on computational results that shows good agreement between the modelprediction and observed results.

\section{PART IV. EXPERIMENTAL APPROACHES}

Various experimental approaches in two general categories have been evaluated. Approaches in the first category share a common theme of improving the total drug exposure, by enhancing the delivery of agents to bladder tissues (e.g., using permeation enhancers, iontophoresis/electrophoresis, hyperthermia, disrupting the extracellular matrix), prolonging the exposure (e.g., using bioadhesive), or enhancing cell membrane permeability (intravesical hyperthermia). The second category is gene therapy, with the goal of either correcting the mutated and malfunctioned genes responsible for tumor formation and progression or as a means to deliver intrinsic or extrinsic signals for cell destruction.

Permeation enhancers. Approaches to enhance urothelium permeability or drug transport in extracellular matrix include chemical and physical methods, as follows.

The chemical methods use permeation enhancers such as dimethyl sulfoxide (DMSO), which has been used to treat interstitial cystitis (79). Co-administration of DMSO (10$50 \%$ ) promoted the penetration of water-soluble drugs (e.g., cisplatin, pirarubicin, doxorubicin) (80-82) and a lipophilic drug paclitaxel (83) across the urothelium in dogs or rats. However, DMSO also promoted the urine production rate and the absorption of paclitaxel from bladder tissues into systemic circulation, thereby partially negating the benefits of improved drug partition into bladder tissues (83).

Other permeability enhancers have been evaluated. Chitosan is a cationic polysaccharide that, by binding to the negative charges on cell membrane, causes rearrangement of cellular tight junctions and enhances paracellular drug transport. Polycarbophil is a mucoadhesive polyacrylic acid cross-linked with divinyl glycol that, by chelating with extracellular calcium ions, causes opening of cellular tight junctions. In isolated porcine bladder in vitro, co-administration of chitosan $(0.05 \%$ to $1 \% \mathrm{w} /$ $\mathrm{v})$ or polycarbophil $(1 \% \mathrm{w} / \mathrm{v})$ promoted the tissue penetration of moxifloxacin (pKa values of 6.4 and 9.5) or pipemidic acid ( $\mathrm{pKa}$ values 5.4 and 8.2 ) by 3 - or 4 -fold $(84,85)$.

Hyaluronan or hyaluronic acid, a glycosaminoglycan, is a major component of extracellular matrix. Hyaluronidase, an enzyme that hydrolyzes the hyaluronan network, can be safely administered to humans and has been approved for treating vitreous hemorrhage $(86,87)$. Co-administration of hyaluronidase improves drug diffusion into bladder mucosa in rats (88). In humans, hyaluronidase did not improve the efficacy of cisplatin in 33 patients (89), but significantly 
reduced the disease recurrence in superficial bladder cancer patients compared to MMC alone ( $7 \%$ in 43 patients versus $32 \%$ in 63 patients) without enhancing the systemic drug absorption (90,91). Hyaluronidase has other interesting properties; it acts as either a tumor suppressor or promoter depending on the cell type and concentration (92,93). Low concentration of hyaluroindase (14-40 milliunits $/ 10^{6}$ cells) stimulated tumor growth whereas high concentration (>100 milliunits $/ 10^{6}$ cells) induced apoptosis and inhibited tumor formation. These properties indicate the need of carefully fine-tuning the hyaluronidase treatment conditions.

Physical methods to disrupt the urothelium include electromotive therapy (iontophoresis/electrophoresis) and hyperthermia. Both methods are under clinical evaluations. Electromotive administration, by temporary breaching of the urothelium, increases the transport of MMC across urothelium in human bladders (six to nine times higher compared to MMC alone) and absorption into the plasma ( $\sim 5$ times higher peak plasma concentration); the peak concentration was $43 \mathrm{ng} / \mathrm{ml}$ or about $1 / 10$ of the threshold toxic concentration of $400 \mathrm{ng} / \mathrm{ml}$ $(94,95)$. An earlier study in 28 patients with superficial bladder tumors suggests that adding electromotive administration to intravesical MMC did not improve the complete response rate at 10 weeks post-treatment (40\% for electromotive MMC vs $41 \%$ for MMC alone), but improved he reduction in the recurrence rate (from $60 \%$ to $33 \%$ ) and the duration of diseasefree interval (from 10.5 to 14.5 months) (96). A more recent three-arm study compared MMC alone, MMC plus electromotive administration, and BCG in 117 high risk patients. The results show that, compared to MMC alone, electromotive MMC significantly improved the recurrence-free rate $(58 \% \mathrm{vs}$ $31 \%$ at 6 months, $p=0.012$ ) and significantly prolonged the median time to recurrence (35 vs 19.5 months, $p=0.013$ ) (97). The combination of $\mathrm{MMC}$ and electromotive administration produced similar benefits as BCG (64\% recurrence-free at 6 months and median time to recurrence of 266 months, $p>$ $0.05)$. Another recent randomized trial in 212 high risk patients further demonstrated a greater efficacy for a combination of BCG followed by MMC plus electromotive administration, compared to single agent BCG (prolongation of disease-free interval from 21 to 69 months, reduction of recurrence rate from 57.9 to $41.9 \%$, reduction of progression rate from 21.9 to $9.3 \%$, decrease of overall death rate from all causes from 32.4 to $21.5 \%$ and decrease of disease-specific death rate from 16.2 to $5.6 \%$ ) (98). These results compare favorably to the earlier data that showed no benefits in combining MMC and BCG relative to single agents (99-102).

Intravesical thermo-chemotherapy, combination of chemotherapy and localized hyperthermia, is under clinical evaluation. Hyperthermia enhances the effects of chemotherapy on inhibition of DNA synthesis and DNA damage, increases cell membrane permeability and alters intracellular drug trafficking and distribution (103-105). Intravesical hyperthermia is delivered using a microwave applicator inserted inside the bladder cavity, where the bladder wall temperature is maintained at $42-$ $45^{\circ} \mathrm{C}$. The drug solution is recycled to avoid overheating. In two single arm trials in intermediate-to-high risk patients, this modality showed a recurrence-free rate of $91 \%$ after a mean follow up of 289 days ( $n=22)(105), 86 \%$ at 1 year and $75 \%$ at 2 year $(n=90)(106)$. A multi-center, randomized trial compar- ing the combination of intravesical MMC plus hyperthermia with MMC alone in superficial bladder cancer patients shows that adding hyperthermia improved the recurrence-free rate at 24 months $(n=83,83 \%$ vs $43 \%, p=0.0002)$ (23).

Prolonging residence time. Intravesical therapy is usually given over $2 \mathrm{hr}$, after which time the drug is drained from the bladder. Sustained-retention delivery platforms such as bioadhesive microspheres or hydrogel systems can serve as drug depots, thereby extending the drug exposure in the bladder cavity beyond the voiding of urine. In a mouse bladder cancer model, bioadhesive, paclitaxel-loaded poly (methylidene malonate) microspheres were retained on bladder wall for more than $48 \mathrm{hr}$, and yielded survival advantage over a solution of paclitaxel in 5\% Tween 80 (9-week survival rate of 91\% vs 58\%) (107). Similarly, a solution-state thermosensitive poly-(ethylene glycol)-poly (lactic acid-co-glycolic acid)-poly (ethylene glycol) polymer, capable of transforming itself into hydrogel matrix at body temperature $\left(37^{\circ} \mathrm{C}\right)$, showed sustained drug release/ retention in rat bladders over multiple bladder voidings for up to $24 \mathrm{hr}$ (108). Ye et al reported a gelatin-doxorubicin complex that released doxorubicin for up to12 days (109). None of the above formulations have been evaluated in humans.

Magnetic targeting. Magnetic targeting uses a magnet placed externally on the skin covering a predetermined site in the bladder (typically where tumors reside) to localize drugcontaining magnetic particles in tumors, thereby providing continuous exposure to high drug concentrations in tumors. In swine bladders, administration of magnetic doxorubicinloaded microparticles (10 to $80 \mathrm{mg}$ drug in $300-800 \mathrm{mg}$ magnetic particles) followed by 30 -min of external magnetic targeting yielded localization of microparticles in superficial and deep tissue layers of the magnetic-targeted sites (primarily in superficial submucosa) that were retained for at least 44 days (110).

Gene therapy. Gene therapy poses several theoretical advantages over chemotherapy: (a) high selectivity for tumor cells with mutated genes, (b) restore cell growth to normalcy by correcting genetic defects rather than by killing cells, and (c) avoiding the emergence of chemoresistance. Development of gene therapy in bladder cancer has focused on modifying the mutated urothelial cells and restoring normal functions of tumor suppressor genes. A popular target is p53. Mutation of p53 gene or loss of p53 functions leads to uncontrolled cell growth (111). As p53 mutation is found in about $40 \%$ of bladder cancer patients with advanced transitional carcinoma (112), p53 gene therapy is an attractive therapeutic approach.

The two major types of gene delivery systems are viral and nonviral vectors. Viral vectors include adenovirus and vaccinia. The advantages of adenoviral vector include: (a) it is not integrated into host chromosomes, (b) ease of obtaining recombinant proteins and producing vectors in high titers, and (c) high viability of host cells post-infection. Adenovirus normally enters a cell via its membrane receptor, coxsackie/ adenovirus receptor (CAR) $(113,114)$. The luminal surface of the urothelium is coated with a glycosaminoglycans (GAG) layer (115), which retards the adherence of adenoviral vectors to CAR and the subsequent gene transfection (116). The GAG layer can be disrupted by ethanol (117), surfactants (e.g., dodecyl-beta-D-maltoside or sodium dodecyl sulfate) (118), Syn3 (a synthetic polyamide) (119) and low concentra- 
tion of hydrochloric acid (60 mM). Hydrochloric acid was able to promote the transfection efficiency of intravesical E. Coli LacZ gene in adenoviral vector from $10 \%$ to $80 \%$ in rat urothelial cells (120). Syn3 acts as a transfer enhancer that circumvents the need for CAR binding (119).

A comparison of intravesical instillation (single administration) of viral IFN gene therapy (Ad-IFN/Syn3, recombinant replication-deficient adenovirus containing human IFN alpha-2b gene and Syn3) to intravesical IFN showed that IFN gene therapy resulted in much higher IFN concentrations (1000-times higher peak level) that were sustained for a much longer duration (96 hr or longer vs undetectable at $24 \mathrm{hr}$ ) in urothelial tissues (121). This same study further showed that a second dose of viral gene therapy on day 3 was sufficient to maintain the IFN level in urine. Another study demonstrated that intravesical treatment with Ad-IFN/Syn3 for $1 \mathrm{hr}$ on two consecutive days resulted in more than $40 \%$ shrinkage of murine bladder tumors; the extent of antitumor effect was correlated with the dose size of Ad-IFN and the IFN concentration in urine (122). Other replication-deficient adenovirus-mediated gene therapy targeting p53 (123), bFGF (124), IL-8 (125) also demonstrated successful gene delivery and effective inhibition of tumor growth in orthotopic (123) or subcutaneous $(124,125)$ mouse bladder tumor models.

Another approach is the replication-competent oncolytic adenovirus, which, through self replication, results in lysis of tumor cells. CG0070, an oncolytic adenovirus encoding granulocyte macrophage colony stimulating factor (GMCSF), preferentially replicates in retinoblastoma proteindefective bladder cancer cells and produces GM-CSF that activates host immune response (126). The tumor selectivity of CG0070 was indicated by the 100 -fold higher replication and 1000-fold greater cytotoxicity in bladder transitional cell carcinoma cells compared to normal cells. CG0070 showed significant activities against orthotopic and subcutaneous human xenograft bladder tumor model in mice (126), and is undergoing phase 1 clinical trial in patients with recurrent bladder cancer after BCG treatment (127).

Vaccinia is a large, double-stranded DNA virus used for vaccination against smallpox (128). The advantages of recombinant vaccinia virus ( $\mathrm{rVV}$ ) include application in a wide range of hosts, rapid infection and efficient expression of inserted transgenes (112). Early experiments demonstrated that rVV effectively introduced functional genes into bladder tumor cells in vitro and in vivo (129). In a syngeneic, orthotopic mouse bladder tumor model, delivery of p53 gene using rVV (rVV-TK-53) resulted in 33\% survival on day 70, whereas the delivery using phosphate buffer or empty vector showed no survival benefits (112).

Several phase I trials demonstrated that intravesical gene therapy can be safely administered to bladder cancer patients; no dose-limiting toxicity was observed for Dryvax vaccinia viral vectors (no therapeutic gene, 4 patients) (130) and adenoviral vector containing wild-type p53 gene with a gene-transfer enhancer (Big CHAP, 12 patients, single treatment) (131). The latter treatment resulted in the induction of RNA and protein levels of p21/WAF1 (downstream target of p53) in patient tumors, and the expression of vector DNA uniformly throughout the urothelium and submucosal tumors, indicating successful transgene expression. A third study in 13 patients receiving repeated doses of adenoviral vector-mediated p53 (Ad5CMV- p53) on a 28-day cycle also showed good tolerability at the highest dose of $10^{12}$ viral particles (132). Specific transgene expression was observed in 2 of 7 patients that yielded biopsy samples. Two recently initiated studies are evaluating intravesical Ad-IFN/Syn3 (133) and Ad-GM-CSF (127) in patients with recurrent, BCG-refractory bladder cancer.

In intravesical viral gene delivery, the primary safety concern is the inadvertent absorption of the vectors into the systemic circulation, as viral vectors may produce excessive immune response or insertional mutagenesis in host cells. In comparison, nonviral vectors (e.g., Lipoplexes) have the advantages of simple preparation, low-cost, easy manufacturing, low immunogenicity, and can carry larger size genes (134). In mice bearing established orthotopic bladder tumors, intravesical treatments of IL-2 gene therapy (delivered in cationic liposomal formulations, 6 treatments given every other day) resulted in higher survival rate, compared to the reporter gene control (40\% versus $0 \%$ on day 60 ) (135). Similarly, IL-12 gene therapy (delivered in cationic liposomes, 6 treatments given every 3 days) was equally effective as high dose BCG $(400 \mu \mathrm{g})$ in the same mouse model, and both treatments were significantly better compared to the reporter gene control (30\% survival on day 60 vs $0 \%$ ). Interestingly, IL-12 gene therapy was more efficacious than BCG in the surviving mice that were again implanted with orthotopic bladder tumors $(100 \%$ vs $0 \%$ survival on day 60 after the reimplantation of tumor cells), suggesting more durable benefits of IL-12 gene therapy (136). Lipid-based gene vectors have undergone clinical evaluation in other cancer types, with good safety records (137-143).

Other experimental approaches. Small interfering RNA (siRNA) is emerging as novel cancer therapeutic agents (144146). siRNA silences the expression of the targeted gene in a sequence-specific manner on the mRNA level. Treatment with survivin- or telomerase-targeted siRNA down-regulated the expression of the corresponding protein and suppressed the growth of bladder tumor cells (147-149). Instillation of siRNA/cationic liposomes targeting polo-like kinase-1 or PLK-1, a biomarker of poor prognosis in a murine orthotopic bladder cancer model, resulted in significant inhibition of PLK-1 expression and lowering the PLK-1 protein level in bladder tissues, in a time- and dose-dependent manner (150).

Another emerging experimental approach, still in preclinical evaluation, is using chemosensitizer to enhance the activity of agents with demonstrated clinical benefits. In cultured cells, meglumine-modified eicosapentaenoic acid, an omega-3 fatty acid, enhances the cellular uptake and activity of epirubicin and MMC (151). Our laboratory has shown that suramin, when given at non-cytotoxic doses, enhances the activity of MMC against human transitional cell RT4 xenograft tumor in mice (152). Suramin is a polyanion with over 20 molecular targets, including growth factors and proteins involved in signaling pathways important to cell cycle check points (153).

\section{PART V. PERSPECTIVES}

Because of the unique anatomical and physiological properties of the urinary bladder, including easy access for 
instilling treatment and limited systemic absorption of the instilled agent, delivery of effective concentrations of therapeutics to bladder tumors during intravesical therapy is more readily accomplished compared to tumors located in systemic organs (e.g., lungs). From this perspective, treatment of bladder cancer bears similarity to treating tumor cells under in vitro conditions. Hence, disease eradication or cures are real possibilities and should be the goal of future translational research. In addition, as shown in our work on intravesical MMC therapy, the computational drug transport models enable the prediction of drug concentrations in different parts of the bladder in vivo and, together with the pharmacodynamic data in human bladder tumors, can be used to predict the outcomes of different treatment conditions (e.g., dose, concentration, dwell time) and thereby enable the selection of optimal treatment conditions. From the standpoint of clinical therapy development, such computational approaches enable the projection of the anticipated benefits, including the margins of errors, of the experimental treatments and consequently the selection of appropriate patient sample sizes for detecting the desired statistical significance. Such quantitative approaches have the potential of improving the success rate of the clinical development of new treatments, which currently stands at $<6 \%$ for cancer therapeutics (154). Finally, additional lessons learned from the use of intravesical therapy may improve the utility of other intracavity or regional therapies such as intraperitoneal treatment of peritoneal cancers or intrathecal treatment of brain cancer.

\section{ACKNOWLEDGEMENTS}

This work was supported in part by a research grant R21CA111770 from the National Cancer Institute, NIH, DHHS.

\section{REFERENCES}

1. American Cancer Society. Cancer facts and figures. American Cancer Society, Atlanta, GA, 2007.

2. E. A. Schenk-Braat, and C.H. Bangma. Immunotherapy for superficial bladder cancer. Cancer Immunol. Immunother. 54:414-423 (2005).

3. E. A. De Bruijn, H. P. Sleeboom, P. J. van Helsdingen, A. T. van Oosterom, U. R. Tjaden, and R. A. Maes. Pharmacodynamics and pharmacokinetics of intravesical mitomycin $\mathrm{C}$ upon different dwelling times. Int. J. Cancer. 51:359-364 (1992).

4. M. Chai, M. G. Wientjes, R. A. Badalament, J. K. Burgers, and J. L. Au. Pharmacokinetics of intravesical doxorubicin in superficial bladder cancer patients. J. Urol. 152:374-378 (1994).

5. D. Song, M. G. Wientjes, and J. L. Au. Bladder tissue pharmacokinetics of intravesical taxol. Cancer Chemother. Pharmacol. 40:285-292 (1997).

6. U. Engelmann, H. J. Knopf, and J. Graff. Interferon-alpha $2 b$ instillation prophylaxis in superficial bladder cancer-a prospective, controlled three-armed trial. Project Group BochumInterferon and Superficial Bladder Cancer. Anti-cancer Drugs. 3(Suppl 1):33-37 (1992).

7. N. E. Stavropoulos, K. Hastazeris, I. Filiadis, I. Mihailidis, E. Ioachim, Z. Liamis et al. Intravesical instillations of interferon gamma in the prophylaxis of high risk superficial bladder cancer -results of a controlled prospective study. Scand. J. Urol. Nephrol. 36:218-222 (2002).

8. F. N. Joudi, B. J. Smith, and M. A. O'Donnell. Final results from a national multicenter phase II trial of combination bacillus Calmette-Guerin plus interferon alpha-2B for reducing recurrence of superficial bladder cancer. Urol. Oncol. 24:344348 (2006).

9. A. M. Nouri, R. Hyde, and R. T. Oliver. Clinical and immunological effect of intravesical interleukin-2 on superficial bladder cancer. Cancer Immunol. Immunother. 39:68-70 (1994).

10. G. Ferlazzo, C. Magno, R. Iemmo, M. Rizzo, G. Lupo, C. Semino et al. Treatment of superficial bladder cancer with intravesical perfusion of rIL-2: a follow-up study. Anticancer Res. 16:979-980 (1996).

11. C. Magno, D. Melloni, A. Gali, G. Mucciardi, G. Nicocia, B. Morandi et al. The anti-tumor activity of bacillus CalmetteGuerin in bladder cancer is associated with an increase in the circulating level of interleukin-2. Immunol. Lett. 81:235-238 (2002).

12. S. K. Clinton, E. Canto, and M. A. O'Donnell. Interleukin-12. Opportunities for the treatment of bladder cancer. Urol. Clin. North Am. 27:147-155 (2000).

13. G. R. Weiss, M. A. O'Donnell, K. Loughlin, K. Zonno, R. J. Laliberte, and M. L. Sherman. Phase 1 study of the intravesical administration of recombinant human interleukin-12 in patients with recurrent superficial transitional cell carcinoma of the bladder. J. Immunother. (1997). 26:343-348 (2003).

14. V. Serretta, G. Corselli, B. Piazza, C. R. Franks, P. A. Palmer, G. J. Roest et al. Intravesical therapy of superficial bladder transitional cell carcinoma with tumor necrosis factor-alpha: preliminary report of a phase I-II study. Eur. Urol. 22:112-114 (1992).

15. V. Serretta, B. Piazza, C. Pavone, S. Piazza, and M. PavoneMacaluso. Is there a role for recombinant tumor necrosis factor alpha in the intravesical treatment of superficial bladder tumors?-a phase II study. Int. J. Urol. 2:100-103 (1995).

16. D. B. Glazier, R. R. Bahnson, D. G. McLeod, R. W. von Roemeling, E. M. Messing, and M.S. Ernstoff. Intravesical recombinant tumor necrosis factor in the treatment of superficial bladder cancer: an Eastern Cooperative Oncology Group study. J. Urol. 154:66-68 (1995).

17. M. M. Wishahi, I. M. Ismail, H. Ruebben, and T. Otto. Keyhole-limpet hemocyanin immunotherapy in the bilharzial bladder: a new treatment modality? Phase II trial: superficial bladder cancer. J. Urol. 153:926-928 (1995).

18. C. D. Jurincic-Winkler, K. A. Metz, J. Beuth, and K. F. Klippel. Keyhole limpet hemocyanin for carcinoma in situ of the bladder: a long-term follow-up study. Eur. Urol. 37(Suppl 3):45-49 (2000).

19. T. M. de Reijke, E. C. de Boer, D. H. Schamhart, and K. H. Kurth. Immunostimulation in the urinary bladder by local application of Nocardia rubra cell wall skeleton preparation (Rubratin) for superficial bladder cancer immunotherapy-a phase I/II study. Urol. Res. 25:117-120 (1997).

20. E. C. de Boer, T. M. De Reijke, P. C. Vos, K. H. Kurth, and D. H. Schamhart. Immunostimulation in the urinary bladder by local application of Nocardia rubra cell-wall skeletons (Rubratin) and bacillus Calmette-Guerin as therapy for superficial bladder cancer: a comparative study. Clin. Infect. Dis. 31(Suppl 3):S109-S114 (2000).

21. A. Allona Moncada, S. Garcia Vaquero, A. Zuloaga Gomez, J. L. Martinez Torres, R. Lopez-Pardo, J. Molina et al. Preliminary results of a multicenter study with mitomycin $\mathrm{C}$ in superficial bladder tumors (Ta, T1). Actas Urol. Esp. 12:424429 (1988).

22. A. P. van der Meijden, and F. M. DeBruyne. Treatment schedule of intravesical chemotherapy with mitomycin $\mathrm{C}$ in superficial bladder cancer: short-term courses or maintenance therapy. Urology. 31(Suppl 3):26-29 (1988).

23. R. Colombo, A. Lev, L. F. Da Pozzo, M. Freschi, G. Gallus, and P. Rigatti. A new approach using local combined microwave hyperthermia and chemotherapy in superficial transitional bladder carcinoma treatment. J. Urol. 153:959-963 (1995).

24. J. Kondas, L. Kiss, A. Hatar, A. Kiss, T. Lukacs, P. Szeldeli et al. The effect of intravesical mitomycin $\mathrm{C}$ on the recurrence of superficial (Ta-T1) bladder cancer. A Hungarian Multicenter Study. Int. Urol. Nephrol. 31:451-456 (1999).

25. S. M. Di Stasi, A. Giannantoni, R. L. Stephen, G. Capelli, P. Navarra, R. Massoud et al. Intravesical electromotive mitomycin $\mathrm{C}$ versus passive transport mitomycin $\mathrm{C}$ for high risk 
superficial bladder cancer: a prospective randomized study. $J$. Urol. 170:777-782 (2003).

26. K. Obata, Y. Ohashi, H. Akaza, S. Isaka, S. Kagawa, K. Koiso et al. Prophylactic chemotherapy with intravesical instillation of adriamycin and oral administration of 5-fluorouracil after surgery for superficial bladder cancer. The Japanese Urological Cancer Research Group for Adriamycin. Cancer Chemother. Pharmacol. 35:S88-S92 (1994).

27. K. Ueda, H. Sakagami, Y. Masui, and T. Okamura. Single instillation of hydroxypropylcellulose-doxorubicin as treatment for superficial bladder carcinoma. Cancer Chemother. Pharmacol. 35:S81-83 (1994).

28. S. Hinotsu, H. Akaza, S. Isaka, S. Kagawa, K. Koiso, T. Kotake et al. Intravesical instillation of doxorubicin or epirubicin for chemoprophylaxis of superficial bladder cancer-the fifth study of the Japanese Urological Cancer Research Group for Adriamycin/Farumorubicin. Gan To Kagaku Ryoho. 29:73-80 (2002).

29. N. Gad el Mawla, M. A. Mansour, S. Eissa, N. M. Ali, I. Elattar, M. R. Hamza et al. A randomized pilot study of high-dose epirubicin as neoadjuvant chemotherapy in the treatment of cancer of the bilharzial bladder. Ann. Oncol. 2:137-140 (1991).

30. W. G. Jones, M. P. Sokal, M. J. Ostrowski, and D. W. Newling. A phase II study of epirubicin in advanced transitional cell bladder cancer. The Yorkshire Urological Cancer Research Group. Clin. Oncol. 5:25-29 (1993).

31. K. Nomata, M. Noguchi, H. Kanetake, N. Tsuda, M. Hayashi, S. Yamashita et al. Intravesical adjuvant chemotherapy for superficial transitional cell bladder carcinoma: results of a randomized trial with epirubicin comparing short-term versus long-term maintenance treatment. Cancer Chemother. Pharmacol. 50:266270 (2002).

32. B. Liu, Z. Wang, B. Chen, J. Yu, P. Zhang, Q. Ding et al. Randomized study of single instillation of epirubicin for superficial bladder carcinoma: long-term clinical outcomes. Cancer Invest. 24:160-163 (2006).

33. Y. Horn, A. Eidelman, N. Walach, E. Yuval, and A. Markowitz. Treatment of superficial bladder tumors in a controlled trial with thio-TEPA versus adriamycin. J. Surg. Oncol. 27:67-69 (1984).

34. J. A. Martínez-Piñeiro, J. Jiménez León, L. Martínez-Piñeiro Jr, L. Fiter, J. A. Mosteiro, J. Navarro et al. Bacillus CalmetteGuerin versus doxorubicin versus thiotepa: a randomized prospective study in 202 patients with superficial bladder cancer. J. Urol. 143:502-506 (1990).

35. J. Flamm, W. Kühnel, and S. Dona. Short-term versus long-term chemoprevention of recurrence with Epodyl following transurethral resection of superficial urinary bladder cancer. Urologe A. 28:99-102 (1989).

36. J. Flamm, G. Donner, A. Bucher, W. Holtl, W. Albrecht, and L. Havelec. Topical immunotherapy (KLH) vs. chemotherapy (Ethoglucid) in prevention of recurrence of superficial bladder cancer. A prospective randomized study. Urologe A. 33:138-143 (1994).

37. K. Kurth, U. Tunn, R. Ay, F. H. Schröder, M. Pavone-Macaluso, F. Debruyne et al. Adjuvant chemotherapy for superficial transitional cell bladder carcinoma: long-term results of a European Organization for Research and Treatment of Cancer randomized trial comparing doxorubicin, ethoglucid and transurethral resection alone. J. Urol. 158:378-384 (1997).

38. D. W Newling, J. Hetherington, S. K. Sundaram, M. R. Robinson, and L. Kisbenedek. The use of valrubicin for the chemoresection of superficial bladder cancer-a marker lesion study. Eur. Urol. 39:643-647 (2001).

39. A. L. Patterson, R. E. Greenberg, L. Weems, R. Bahnson, Z. Wajsman, M. Israel et al. Pilot study of the tolerability and toxicity of intravesical valrubicin immediately after transurethral resection of superficial bladder cancer. Urology. 56:232235 (2000).

40. G. Steinberg, R. Bahnson, S. Brosman, R. Middleton, Z. Wajsman, and M. Wehle. Efficacy and safety of valrubicin for the treatment of Bacillus Calmette-Guerin refractory carcinoma in situ of the bladder. The Valrubicin Study Group. J. Urol. 163:761-767 (2000).

41. S. D. Williams, L. H. Einhorn, and J. P. Donohue. cis-Platinum combination chemotherapy of bladder cancer: an update. Cancer Clin. Trials. 2:335-338 (1979).
42. J. A. Martinez-Piñeiro, M. Gonzalez Martin, F. Arocena, N. Flores, C. R. Roncero, J. A. Portillo et al. Neoadjuvant cisplatin chemotherapy before radical cystectomy in invasive transitional cell carcinoma of the bladder: a prospective randomized phase III study. J. Urol. 153:964-973 (1995).

43. N. K. Gogna, J. H. Matthews, S. L. Turner, H. Mameghan, G. M. Duchesne, N. Spry et al. Efficacy and tolerability of concurrent weekly low dose cisplatin during radiation treatment of localised muscle invasive bladder transitional cell carcinoma: a report of two sequential Phase II studies from the Trans Tasman Radiation Oncology Group. Radiother. Oncol. 81:9-17 (2006).

44. W. M. Stadler, T. Kuzel, B. Roth, D. Raghavan, and F. A. Dorr Phase II study of single-agent gemcitabine in previously untreated patients with metastatic urothelial cancer. J. Clin. Oncol. 15:3394-3398 (1997).

45. V. Lorusso, C. F. Pollera, M. Antimi, G. Luporini, C. Gridelli, G. L. Frassineti et al. A phase II study of gemcitabine in patients with transitional cell carcinoma of the urinary tract previously treated with platinum. Italian Co-operative Group on Bladder Cancer. Eur. J. Cancer. 34:1208-1212 (1998).

46. H. M. Khaled, M. R. Hamza, O. Mansour, R. Gaafar, and M. S. Zaghloul. A phase II study of gemcitabine plus cisplatin chemotherapy in advanced bilharzial bladder carcinoma. Eur. J. Cancer. 36(Suppl 2):34-37 (2000).

47. E. M. Uchio, W. M. Linehan, W. D. Figg, and M. M. Walther. A phase I study of intravesical suramin for the treatment of superficial transitional cell carcinoma of the bladder. J. Urol. 169:357-360 (2003)

48. J. J. Ord, E. Streeter, A. Jones, K. Le Monnier, D. Cranston, J. Crew et al. Phase I trial of intravesical Suramin in recurrent superficial transitional cell bladder carcinoma. Br. J. Cancer. 92:2140-2147 (2005).

49. P. A. Kosmidis, C. Bacoyiannis, and H. Hatzichristou. Combination chemotherapy with cis-platinum, adriamycin, mitomycin$\mathrm{C}$ (PAM) in patients with advanced bladder cancer. J. Chemother. 2:397-400 (1990).

50. C. Sola, J. Mallafre, L. Mendoza Solorzano, A. Segarra, M. Daniels, N. Vinolas et al. Carboplatin, methotrexate, vinblastine and epirubicin (Carbo-MVE) for transitional cell bladder carcinoma. Ann. Oncol. 4:313-316 (1993).

51. W. K. Oh, J. Manola, J. P. Richie, K. R. Loughlin, and P. W. Kantoff. A phase II trial of methotrexate, cisplatin, 5-fluorouracil, and leucovorin in the treatment of invasive and metastatic urothelial carcinoma. Cancer. 86:1329-1334 (1999).

52. T. H. Ecke, P. Bartel, S. Koch, J. Ruttloff, and F. Theissig. Chemotherapy with gemcitabine, paclitaxel, and cisplatin in the treatment of patients with advanced transitional cell carcinoma of the urothelium. Oncol. Rep. 16:1381-1388 (2006).

53. S. B. Malkowicz. The role of intravesical chemotherapy in the treatment of bladder cancer. In S. P. Lerner, M. Schoenberg, and C. Sternberg (eds.), Textbook of Bladder Cancer, Informa Healthcare, London, 2006, pp. 335-340.

54. L. A. Pinke, and D. L. Lamm. Intravesical immunotherapy: BCG. In S. P. Lerner, M. Schoenberg, and C. Sternberg (eds.) Textbook of Bladder Cancer, Informa Healthcare, London, 2006, pp. 353-358.

55. A. Böhle, D. Jocham, and P. R. Bock. Intravesical Bacillus Calmette-Guerin versus mitomycin $\mathrm{C}$ for superficial bladder cancer. A formal meta-analysis of comparative studies on recurrence and toxicity. J. Urol. 169:90-95 (2003).

56. M. A. O'Donnell, J. Krohn, and W. C. DeWolf. Salvage intravesical therapy with interferon-alpha $2 \mathrm{~b}$ plus low dose bacillus Calmette-Guerin is effective in patients with superficial bladder cancer in whom bacillus Calmette-Guerin alone previously failed. J. Urol. 166:1300-1304 (2001).

57. S. P. Punnen, J. L. Chin, and M. A. Jewett. Management of Bacillus Calmette-Guerin (BCG) refractory superficial bladder cancer: results with intravesical BCG and Interferon combination therapy. Can. J. Urol. 10:1790-1795 (2003).

58. J. S. Lam, M. C. Benson, M. A. O'Donnell, A. Sawczuk, A. Gavazzi, M. H. Wechsler et al. Bacillus Calmette-Guerin plus interferon-alpha2B intravesical therapy maintains an extended treatment plan for superficial bladder cancer with minimal toxicity. Urol. Oncol. 21:354-360 (2003). 
59. F. N. Joudi, B. J. Smith, and M. A.O'Donnell. Final results from a national multicenter phase II trial of combination bacillus Calmette-Guerin plus interferon alpha-2B for reducing recurrence of superficial bladder cancer. National BCG-Interferon Phase 2 Investigator Group, Urol. Oncol. 24:344-348 (2006).

60. G. Steinberg, R. Bahnson, S. Brosman, R. Middleton, Z. Wajsman, and M. Wehle. Efficacy and safety of valrubicin for the treatment of Bacillus Calmette-Guerin refractory carcinoma in situ of the bladder. The Valrubicin Study Group. J. Urol. 163:761-767 (2000)

61. G. Dalbagni, P. Russo, B. Bochner, L. Ben-Porat, J. Sheinfeld, P. Sogani et al. Phase II trial of intravesical gemcitabine in Bacille Calmette-Guérin-Refractory transitional cell carcinoma of the bladder. J. Clin. Oncol. 24:2729-2734 (2006).

62. M. Gacci, R. Bartoletti, T. Cai, S. Nerozzi, N. Pinzi, F.Repetti, TUR (Toscana Urologia) Group et al. Intravesical gemcitabine in BCG-refractory T1G3 transitional cell carcinoma of the bladder: a pilot study. Urol. Int. 76:106-111 (2006).

63. M. G. Wientjes, R. A. Badalament, and J. L. Au. Use of pharmacologic data and computer simulations to design an efficacy trial of intravesical mitomycin C therapy for superficial bladder cancer. Cancer Chemother. Pharmacol. 32:255-262 (1993).

64. W. J. Underberg, and H. Lingeman. Aspects of the chemical stability of mitomycin and porfiromycin in acidic solution. $J$. Pharm. Sci. 72:549-553 (1983).

65. J. H. Beijnen, H. Lingeman, H. A. Van Munster, and W. J. Underberg. Mitomycin antitumor agents: a review of their physicochemical and analytical properties and stability. $J$. Pharm. Bio. Anal. 4:275-295 (1986)

66. B. E. Cohen, M. J. Egorin, M. S. Nayar, and P. L. Gutierrez. Effects of $\mathrm{pH}$ and temperature on the stability and decomposition of $N, N$ 'N'-triethylenethiophosphoramide in urine and buffer. Cancer Res. 44:4312-4316 (1984).

67. J. B. Mellett, and L. A. Woods. The comparative physiological disposition of thiotepa and tepa in the dog. Cancer Res. 20:524 532 (1960).

68. K. F. Ilett, R. T. Ong, K. T. Batty, and J. D. Taylor. Effect of urine $\mathrm{pH}$ on the stability of doxorubicin and its recovery from bladder instillations. Br. J. Urol. 65:478-482 (1990).

69. W. C. Yen, T. Schmittgen, and J. L. Au. Different pH dependency of mitomycin $\mathrm{C}$ activity in monolayer and threedimensional cultures. Pharm. Res. 13:1887-1891 (1996).

70. N. M. Harris, P. M. Duffy, T. J. Crook, W. R. Anderson, P. Sharpe, M. C. Hayes et al. Intravesical $\mathrm{pH}$ : a potentially important variable affecting efficacy and the further development of anthracycline chemotherapy for superficial bladder cancer. BJU Int. 90:957-964 (2002).

71. A. M. Cliff, B. Heatherwick, J. Scoble, and N. J. Parr. The effect of fasting or desmopressin before treatment on the concentration of mitomycin $\mathrm{C}$ during intravesical administration. BJU Int. 86:644-647 (2000)

72. M. G. Wientjes, R. A. Badalament, R. C. Wang, F. Hassan, and J. L. Au. Penetration of mitomycin C in human bladder. Cancer Res. 53:3314-3320 (1993).

73. D. Song, M. G. Wientjes, Y. Gan, and J. L. Au. Bladder tissue pharmacokinetics and antitumor effect of intravesical 5-fluorouridine. Clin. Cancer Res. 3:901-909 (1997).

74. M. G. Wientjes, R. A. Badalament, and J. L. Au. Penetration of intravesical doxorubicin in human bladders. Cancer Chemother Pharmacol. 37:539-546 (1996).

75. M. Pavone-Macaluso, N. Gebbia, F. Biondo, S. Bertolini, G. Caramia, and F. P. Rizzo. Permeability of the bladder mucosa to thiotepa, adriamycin, and daunomycin in men and rabbits. Urol. Res. 4:9-13 (1976).

76. G. Lunglmayr, and K. Czech. Absorption studies on intraluminal thio-tepa for topical cytostatic treatment of low-stage bladder tumors. J. Urol. 106:72-74 (1971).

77. I. Grabnar, M. Bogataj, A. Belic, V. Logar, R. Karba, and A. Mrhar. Kinetic model of drug distribution in the urinary bladder wall following intravesical instillation. Int. J. Pharm. 322:52-59 (2006).

78. J. L. Au, R. A. Badalament, M. G. Wientjes, D. C. Young, J. A. Warner, P. L. Venema et al. Methods to improve efficacy of intravesical mitomycin C: results of a randomized phase III trial. J. Natl. Cancer Inst. 93:597-604 (2001).

79. G. R. Sant. Intravesical $50 \%$ dimethyl sulfoxide (Rimso-50) in treatment of interstitial cystitis. Urology. 29(Suppl 4):17-21 (1987).

80. R. H. Schoenfeld, W. D. Belville, W. H. Jacob, A. S. Buck, M. L. Dresner, S. J. Insalaco et al. The effect of dimethyl sulfoxide on the uptake of cisplatin from the urinary bladder of the dog: a pilot study. J. Am. Osteopath. Assoc. 82:570-573 (1983).

81. H. Hashimoto, S. Tokunaka, M. Sasaki, M. Nishihara, and S. Yachiku. Dimethylsulfoxide enhances the absorption of chemotherapeutic drug instilled into the bladder. Urol. Res. 20:233-236 (1992).

82. W. A. See, and Q. Xia. Regional chemotherapy for bladder neoplasms using continuous intravesical infusion of doxorubicin: impact of concomitant administration of dimethyl sulfoxide on drug absorption and antitumor activity. J. Natl. Cancer Inst. 84:510-515 (1992).

83. D. Chen, D. Song, M. G. Wientjes, and J. L. Au. Effect of dimethyl sulfoxide on bladder tissue penetration of intravesical paclitaxel. Clin. Cancer Res. 9:363-369 (2003).

84. M. K. Kos, M. Bogataj, P. Veranic, and A. Mrhar. Permeability of pig urinary bladder wall: time and concentration dependent effect of chitosan. Biol. Pharm. Bull. 29:1685-1691 (2006).

85. I. Grabnar, M. Bogataj, and A. Mrhar. Influence of chitosan and polycarbophil on permeation of a model hydrophilic drug into the urinary bladder wall. Int. J. Pharm. 256:167-173 (2003).

86. B. D. Kuppermann, E. L. Thomas, M. D. de Smet, and L. R. Grillone. Vitrase for Vitreous Hemorrhage Study Groups. Safety results of two phase III trials of an intravitreous injection of highly purified ovine hyaluronidase (Vitrase) for the management of vitreous hemorrhage. Am. J. Ophthalmol. 140:585-597 (2005).

87. (No authors listed). Hyaluronidase (Vitrase)-ISTA: hyaluronidase-ISTA pharmaceuticals. Drugs R\&D. 4:194-97 (2003)

88. M. Sasaki, H. Hashimoto, and S. Yachiku. Studies on enhancement of drug absorption through the bladder mucosa. Nippon Hinyokika Gakkai Zasshi. 85:1353-1362 (1994).

89. Y. Horn, A. Eidelman, N. Walach, M. Waron, and F. Barak. Intravesical chemotherapy of superficial bladder tumors in a controlled trial with cis-platinum versus cis-platinum plus hyaluronidase. J. Surg. Oncol. 28:304-307 (1985).

90. U. Maier, and G. Baumgartner. Mitomycin C plasma levels after intravesical instillation with and without hyaluronidase. $J$. Urol. 135:845-846 (1986).

91. K. Hobarth, U. Maier, and M. Marberger. Topical chemoprophylaxis of superficial bladder cancer with mitomycin $\mathrm{C}$ and adjuvant hyaluronidase. Eur. Urol. 21:206-210 (1992).

92. V. B. Lokeshwar, W. H. Cerwinka, and B. L. Lokeshwar HYAL1 hyaluronidase: a molecular determinant of bladder tumor growth and invasion. Cancer Res. 65:2243-2250 (2005).

93. V. B. Lokeshwar, V. Estrella, L. Lopez, M. Kramer, P. Gomez, M. S. Soloway et al. HYAL1-v1, an alternatively spliced variant of HYAL1 hyaluronidase: a negative regulator of bladder cancer. Cancer Res. 66:11219-11227 (2006).

94. S. M. Di Stasi, G. Vespasiani, A. Giannantoni, R. Massoud, S Dolci, and F. Micali. Electromotive delivery of mitomycin C into human bladder wall. Cancer Res. 57:875-880 (1997).

95. S. M. Di Stasi, A. Giannantoni, R. Massoud, S. Dolci, P. Navarra, G. Vespasiani et al. Electromotive versus passive diffusion of mitomycin $\mathrm{C}$ into human bladder wall: concentration-depth profiles studies. Cancer Res. 59:4912-4918 (1999).

96. M. Brausi, B. Campo, G. Pizzocaro, P. Rigatti, A. Parma, G. Mazza et al. Intravesical electromotive administration of drugs for treatment of superficial bladder cancer: A comparative phase II study. Urology. 51:506-509 (1998).

97. S. M. Di Stasi, A. Giannantoni, R. L. Stephen, G. Capelli, P. Navarra, R. Massoud et al. Intravesical electromotive mitomycin $\mathrm{C}$ versus passive transport mitomycin $\mathrm{C}$ for high risk superficial bladder cancer: a prospective randomized study. $J$. Urol. 170:777-782 (2003).

98. S. M. Di Stasi, A. Giannantoni, A. Giurioli, M. Valenti, G. Zampa, L. Storti et al. Sequential BCG and electromotive mitomycin versus BCG alone for high-risk superficial bladder cancer: a randomised controlled trial. Lancet Oncol. 7:43-51 (2006). 
99. E. Rintala, K. Jauhiainen, P. Rajala, M. Ruutu, E. Kaasinen, O. Alfthan et al. Alternating mitomycin-C and bacillus calmetteguerin instillation therapy for carcinoma in situ of the bladder. J. Urol. 154:2050-2053 (1995).

100. E. Rintala, K. Jauhiainen, E. Kaasinen, M. Nurmi, and O. Alfthan. Alternating mitomycin-C and bacillus calmette-guerin instillation prophylaxis for recurrent papillary (stages Ta-T1) superficial bladder cancer. J. Urol. 156:56-59 (1996).

101. J. A. Witjes, C. T. Caris, N. A. Mungan, F. M. Debruyne, and W. P. Witjes. Results of a randomised phase III trial of sequential intravesical therapy with mitomycin-C and bacillus calmette-guerin $v s$ mitomycin-C alone in patients with superficial bladder cancer. J. Urol. 160:1668-1671 (1998).

102. E. Kaasinen, H. Wijkström, P. U. Malmström, S. Hellsten, M. Duchek, O. Mestad et al. Nordic Urothelial Cancer Group. Alternating mitomycin $\mathrm{C}$ and BCG instillations versus BCG alone in treatment of carcinoma in situ of the urinary bladder: a Nordic study. Eur. Urol. 43:637-645 (2003).

103. P. M. Corry, S. Robinson, and S. Getz. Hyperthermic effects on DNA repair mechanisms. Radiology. 123:475-482 (1977).

104. T. S. Herman, B. A. Teicher, M. Jochelson, J. Clark, G. Svensson, and C. N. Coleman. Rationale for use of local hyperthermia with radiation therapy and selected anticancer drugs in locally advanced human malignancies. Int. J. Hypertherm. 4:143-158 (1988).

105. B. Moskovitz, M. G. eyer, A. Kravtzov, M. Gross, A. Kastin, K. Biton et al. Thermo-chemotherapy for intermediate or high-risk recurrent superficial bladder cancer patients. Ann. Oncol. 16:585-589 (2005).

106. A. G. van der Heijden, L. A. Kiemeney, O. N. Gofrit, O. Nativ, A. Sidi, Z. Leib et al. Preliminary European results of local microwave hyperthermia and chemotherapy treatment in intermediate or high risk superficial transitional cell carcinoma of the bladder. Eur. Urol. 46:65-71 (2004).

107. C. Le Visage, N. Rioux-Leclercq, M. Haller, P. Breton, B. Malavaud, and K. Leong. Efficacy of paclitaxel released from bio-adhesive polymer microspheres on model superficial bladder cancer. J. Urol. 171:1324-1329 (2004).

108. P. Tyagi, Z. Li, M. Chancellor, W. C. De Groat, N. Yoshimura, and L. Huang. Sustained intravesical drug delivery using thermosensitive hydrogel. Pharm. Res. 21:832-837 (2004).

109. Z. Ye, J. Chen, X. Zhang, J. Li, S. Zhou, W. Yang et al. Novel gelatin-adriamycin sustained drug release system for intravesical therapy of bladder cancer. J. Tongji Med. Univ. 21:145148 (2001).

110. T. Leakakos, C. Ji, G. Lawson, C. Peterson, and S. Goodwin. Intravesical administration of doxorubicin to swine bladder using magnetically targeted carriers. Cancer Chemother. Pharmacol. 51:445-450 (2003).

111. R. C. Bast Jr, and G. B. Mills. In J. M. S. Bartlett (ed.), Ovarian Cancer: Methods and Protocols, Humana Press, New Jersey, 2000, pp. 37-48.

112. I. Fodor, T. Timiryasova, B. Denes, J. Yoshida, H. Ruckle, and M. Lilly. Vaccinia virus mediated p53 gene therapy for bladder cancer in an orthotopic murine model. J. Urol. 173:604-609 (2005).

113. J. M. Bergelson, J. A. Cunningham, G. Droguett, E. A. KurtJones, A. Krithivas, J. S. Hong et al. Isolation of a common receptor for coxsackie b viruses and adenoviruses 2 and 5 . Science. 275:1320-1323 (1997).

114. R. P. Tomko, R. Xu, and L. Philipson. HCAR and MCAR, the human and mouse cellular receptors for subgroup $\mathrm{C}$ adenoviruses and group B coxsackie viruses. Proc. Natl. Acad. Sci. U. S. A. 94:3352-3356 (1997).

115. J. D. Lilly, and C. L. Parsons. Bladder surface glycosaminoglycans is a human epithelial permeability barrier. Surg. Gynecol. Obstet. 171:493-496 (1990).

116. J. C. Nickel, J. Downey, A. Morales, L. Emerson, and J. Clark. Relative efficacy of various exogenous glycosaminoglycans in providing a bladder surface permeability barrier. J. Urol. 160:612-614 (1998).

117. H. Engler, S. C. Anderson, T. R. Machemer, J. M. Philopena, R. J. Connor, S. F. Wen et al. Ethanol improves adenovirus-mediated gene transfer and expression to the bladder epithelium of rodents. Urology. 53:1049-1053 (1999).
118. N. Ramesh, B. Memarzadeh, Y. Ge, D. Frey, M. VanRoey, V. Rojas et al. Identification of pretreatment agents to enhance adenovirus infection of bladder epithelium. Mol. Ther. 10:697705 (2004).

119. R. J. Connor, H. Engler, T. Machemer, J. M. Philopena, M. T. Horn, S. Sutjipto et al. Identification of polyamides that enhance adenovirus-mediated gene expression in the urothelium. Gene Ther. 8:41-48 (2001).

120. L. F. Lin, G. Zhu, J. J. Yoo, S. Soker, V. P. Sukhatme, and A. Atala. A system for the enhancement of adenovirus mediated gene transfer to uro-epithelium. J. Urol. 168:813-818 (2002).

121. R. J. Connor, J. M. Anderson, T. Machemer, D. C. Maneval, and $\mathrm{H}$. Engler. Sustained intravesical interferon protein exposure is achieved using an adenoviral-mediated gene delivery system: a study in rats evaluating dosing regimens. Urology. 66:224-229 (2005)

122. Z. Tao, R. J. Connor, F. Ashoori, C. P. Dinney, M. Munsell, J. A. Philopena et al. Efficacy of a single intravesical treatment with Ad-IFN/Syn 3 is dependent on dose and urine IFN concentration obtained: implications for clinical investigation. Cancer Gene Ther. 13:125-130 (2006).

123. P. E. Werthman, K. E. Drazan, J. T. Rosenthal, R. Khalili, and A. Shaked. Adenoviral-p53 gene transfer to orthotopic and peritoneal murine bladder cancer. J. Urol. 155:753-756 (1996).

124. K. Inoue, P. Perrotte, C. G. Wood, J. W. Slaton, P. Sweeney, and C. P. Dinney. Gene therapy of human bladder cancer with adenovirus-mediated antisense basic fibroblast growth factor. Clin. Cancer Res. 6:4422-4431 (2000).

125. K. Inoue, C. G. Wood, J. W. Slaton, T. Karashima, P. Sweeney, and C. P. Dinney. Adenoviral-mediated gene therapy of human bladder cancer with antisense interleukin-8. Oncol. Rep. 8:955964 (2001)

126. N. Ramesh, Y. Ge, D. L. Ennist, M. Zhu, M. Mina, S. Ganesh et al. CG0070, a conditionally replicating granulocyte macrophage colony-stimulating factor-armed oncolytic adenovirus for the treatment of bladder cancer. Clin. Cancer Res. 12:305-313 (2006).

127. Dose-escalation study of CG0070 for bladder cancer after BCG (Bacillus Calmette-Guerin) Failure. (NCI clinical trial protocol \# V-0046, NCT00109655).

128. B. Moss. Reproduction of poxviruses. In H. Frankel-Conrat (ed.), Comprehensive Virology, 3, Plenum Press, New York, 1974, pp. 405-474.

129. S. S. Lee, L. C. Eisenlohr, P. A. McCue, M. J. Mastrangelo, and E. C. Lattime. Intravesical gene therapy: in vivo gene transfer using recombinant vaccinia virus vectors. Cancer Res. 54:33253328 (1994)

130. L. G. Gomella, M. J. Mastrangelo, P. A. McCue, H. C. Maguire Jr, S. G. Mulholland, and E. C. Lattime. Phase I study of intravesical vaccinia virus as a vector for gene therapy of bladder cancer. J. Urol. 166:1291-1295 (2001).

131. J. Kuball, S. F. Wen, J. Leissner, D. Atkins, P. Meinhardt, E. Quijano et al. Successful adenovirus-mediated wild-type p53 gene transfer in patients with bladder cancer by intravesical vector instillation. J. Clin. Oncol. 20:957-965 (2002).

132. L. C. Pagliaro, A. Keyhani, D. Williams, D. Woods, B. Liu, P. Perrotte et al. Repeated intravesical instillations of an adenoviral vector in patients with locally advanced bladder cancer: a phase I study of p53 gene therapy. J. Clin. Oncol. 21:2247-2253 (2003).

133. A phase I study of the safety and tolerability of intravesical administration of SCH 721015 in patients with transitional cell carcinoma of the bladder. MD Anderson cancer center. Clinical trial \# 2005-0580.

134. S. Li, and L. Huang. Nonviral gene therapy: promises and challenges. Gene Ther. 7:31-34 (2000).

135. Y. Horiguchi, W. A. Larchian, R. Kaplinsky, W. R. Fair, and W. D. Heston. Intravesical liposome-mediated interleukin-2 gene therapy in orthotopic murine bladder cancer model. Gene Ther. 7:844-851 (2000).

136. M. Horinaga, K. M. Harsch, R. Fukuyama, W. Heston, and W. Larchian. Intravesical interleukin-12 gene therapy in an orthotopic bladder cancer model. Urology. 66:461-466 (2005).

137. Y. Liao, Y.Y. Zou, W.Y. Xia, and M.C. Hung. Enhanced paclitaxel cytotoxicity and prolonged animal survival rate by a 
nonviral-mediated systemic delivery of E1A gene in orthotopic xenograft human breast cancer. Cancer Gene Ther. 11:594-602 (2004).

138. S. Liu, X. P. Wang, and F. C. Brunicardi. Enhanced cytotoxicity of RIPTK gene therapy of pancreatic cancer via PDX-1 codelivery. J. Surg. Res. 137:1-9 (2007).

139. P. F. Chen, G. F. Fu, H. Y. Zhang, G. X. Xu, and Y. Y. Hou. Liposomal plasmid DNA encoding human thymosin alpha and interferon omega potently inhibits liver tumor growth in ICR mice. J. Gastroenterol. Hepatol. 21:1538-1543 (2006).

140. S. Fletcher, A. Ahmad, E. Perouzel, A. Heron, A. D. Miller, and M. R. Jorgensen. In vivo studies of dialkynoyl analogues of DOTAP demonstrate improved gene transfer efficiency of cationic liposomes in mouse lung. J. Med. Chem. 49:349-357 (2006).

141. E. W. Alton, M. Stern, R. Farley, A. Jaffe, S. L. Chadwick, J. Phillips et al. Cationic lipid-mediated CFTR gene transfer to the lungs and nose of patients with cystic fibrosis: a double-blind placebo-controlled trial. Lancet. 353:947-954 (1999).

142. S. Dow, R. Elmslie, I. Kurzman, G. MacEwen, F. Pericle, and D. Liggitt. Phase I study of liposome-DNA complexes encoding the interleukin-2 gene in dogs with osteosarcoma lung metastases. Hum. Gene Ther. 16:937-946 (2005).

143. C. M. Rudin, J. L. Marshall, C. H. Huang, H. L. Kindler, C. Zhang, D. Kumar et al. Delivery of a liposomal c-raf-1 antisense oligonucleotide by weekly bolus dosing in patients with advanced solid tumors: a phase I study. Clin. Cancer Res. 10:7244-7251 (2004).

144. H. Xia, Q. Mao, H. L. Paulson, and B. L. Davidson. siRNAmediated gene silencing in vitro and in vivo. Nat. Biotechnol. 20:1006-1010 (2002).

145. U. N. Verma, R. M. Surabhi, A. Schmaltieg, C. Becerra, and R. B. Gaynor. Small interfering RNAs directed against $\beta$-Catenin inhibit the in vitro and in vivo growth of colon cancer cells. Clin Cancer Res. 9:1291-1300 (2003).
146. R. M. Schiffelers, A. Ansari, J. Xu, Q. Zhou, Q. Tang, G. Storm et al. Cancer siRNA therapy by tumor selective delivery with ligand-targeted sterically stabilized nanoparticle. Nucleic Acids Res. 32:e149 (2004).

147. S. Ning, S. Fuessel, M. Kotzsch, K. Kraemer, M. Kappler, U. Schmidt et al. SiRNA-mediated down-regulation of survivin inhibits bladder cancer cell growth. Int. J. Oncol. 25:1065-1071 (2004).

148. J. Q. Hou, J. He, X. L. Wang, D. G. Wen, and Z. X. Chen. Effect of small interfering RNA targeting survivin gene on biological behaviour of bladder cancer. Chin. Med. J. 119:17341739 (2006).

149. L. Zou, P. Zhang, C. Luo, and Z. Tu. shRNA-targeted hTERT suppress cell proliferation of bladder cancer by inhibiting telomerase activity. Cancer Chemother. Pharmacol. 57:328-334 (2006).

150. M. Nogawa, T. Yuasa, S. Kimura, M. Tanaka, J. Kuroda, K. Sato et al. Intravesical administration of small interfering RNA targeting PLK-1 successfully prevents the growth of bladder cancer. Clin. Invest. 115:978-985 (2005).

151. S. J. Mackie, D. M. Sharma, A. J. Cooper, N. M. Harris, B. A. Lwaleed et al. Meglumine Eicosapentaenoic acid (MeEPA) a new soluble omega-3 fatty acid formulation: in vitro bladder cancer cytotoxicity tests in combination with epirubicin and mitomycin. Prostaglandins. 75:367-373 (2006).

152. Y. Xin, G. Lyness, D. Chen, S. Song, M. G. Wientjes, and J. L. $\mathrm{Au}$. Low dose suramin as a chemosensitizer of bladder cancer to mitomycin C. J. Urol. 174:322-327 (2005).

153. S. H. Song, M. G. Wientjes, Y. B. Gan, and J. L. Au. Fibroblast growth factors: an epigenetic mechanism of broad spectrum resistance to anticancer drugs. Proc. Natl. Acad. Sci. U. S. A. 97:8658-8663 (2000)

154. P. Nygren, and R. Larsson. Overview of the clinical efficacy of investigational anticancer drugs. J. Intern. Med. 253:46-75 (2003). 